\title{
Perforated Colorectal Cancer
}

\author{
Alexios Tzivanakis, MD, FRCS (Gen Surg) ${ }^{1}$ Brendan J. Moran, MChi, FRCSI ${ }^{1}$ \\ ${ }^{1}$ Peritoneal Malignancy Institute, Basingstoke, Hampshire, \\ United Kingdom \\ Clin Colon Rectal Surg 2020;33:247-252.

\begin{abstract}
Address for correspondence Brendan J. Moran, MChi, FRCSI, Peritoneal Malignancy Institute; The Ark Conference Centre, Dinwoodie Drive, Basingstoke, Hampshire RG24 9NN, United Kingdom (e-mail: Brendan.Moran@hhft.nhs.uk).
\end{abstract}

\begin{abstract}
Keywords

- perforated colorectal cancer

- peritoneal carcinomatosis

- CRS and HIPEC

The majority of patients with colorectal tumors will present via the elective route. However, one-fifth of patients will present as an emergency. The most common cause of emergency presentation of colorectal cancer is obstruction followed by perforation, and in many cases, patients will present with both. We discuss the management of the patient presenting with a perforated colorectal tumor covering the acute presentation and also how to deal with consequences of a perforated tumor, namely, the management of colorectal peritoneal metastasis (CPM). CPM used to be considered a terminal condition; however, a strategy of early detection of CPM, careful patient selection for cytoreductive surgery and hyperthermic intraperitoneal chemotherapy, leads to much improved outcomes and even cure, in some patient compared with systemic chemotherapy alone.
\end{abstract}

\section{Epidemiology}

In the United Kingdom, colorectal cancer is the fourth most commonly diagnosed cancer with 30,710 new patients diagnosed in 2016. ${ }^{1}$ Survival of colorectal cancer is improving over the past decades but is heavily dependent on the stage of cancer at diagnosis. For example, stage I (T1 or T2 N0 M0) colon and rectal cancer has a 5-year survival of more than 90\%, while stages III (T1-4 N1-2 M0) and IV (any T any N but M1) 63 and 7\%, respectively. ${ }^{2}$

The majority of patients (55\%) present via the elective route (symptomatic patient presenting to their general practitioner/ family physician) and $\sim 9 \%$ via the national screening program. About $20 \%$ of patients will present as an emergency to the acute surgical services. The most common reason $(8-29 \%)^{3}$ for an emergency presentation is obstructive symptoms, such as inability to pass stool or flatus, abdominal pain, distention, and/or vomiting. In $\sim 19 \%$ of these cases, the bowel will have had perforated, either at the tumor site due to direct tumor growth/necrosis or proximal to the obstructing tumor, usually, but not exclusively, a cecal perforation. ${ }^{4}$

Overall, $\sim 29.4 \%$ of the emergency and $22.2 \%$ of the elective/screening patients have T4 disease at presentation. ${ }^{1}$ Patients presenting as an emergency have a significantly worse prognosis compared with those treated electively. ${ }^{5}$

Issue Theme Novel Treatment Strategies for Advanced and Aggressive Colorectal Cancer-Multidisciplinary Solutions; Venkatesh Munikrishnan, MRCS (Ed), FRCS(Ed), CCT(UK), and Manish Chand, MBBS, BSC, FRCS, FASCRS, MBA, PhD

\section{Perforated Colorectal Cancer}

About 2 to $12 \%$ of patient with colorectal disease present with perforation making it the second most common emergency presentation of colorectal cancer patients. ${ }^{6}$ The perforation can result in either free spillage of bowel contents and tumor cells into the peritoneal cavity or may be contained within an abscess cavity. Patients with free perforation tend to have worse outcomes as they have significantly abnormal physiology and usually rapidly progress to peritonitis and septic shock with all its critical sequelae. Perioperative mortality rates are high (around 12\%) despite recent advances in intensive care medicine and perioperative management of the critically unwell surgical patient. Interestingly, in the United Kingdom, data from the 2017 National Emergency Laparotomy Audit show a similar mortality rate for all patients undergoing emergency laparotomy for all indications of $\sim 13$ to $18 \%{ }^{8}$ This observation suggests that patients with perforation secondary to malignancy do not die because of their malignant process but rather from the associated physiological challenges of sepsis. It is therefore very important that the priority on treating these patients is the same as of any surgical patient presenting with peritonitis and sepsis. In 2008, Dellinger et $\mathrm{al}^{9}$ proposed the "surviving sepsis guidelines" and the implantation of the "Sepsis 6" bundle within an hour of diagnosis of
Copyright $\odot 2020$ by Thieme Medical Publishers, Inc., 333 Seventh Avenue, New York, NY 10001, USA. Tel: +1(212) 760-0888.
DOI https://doi.org/ 10.1055/s-0040-1713741. ISSN 1531-0043. 
sepsis. These measures have been widely adopted in the United Kingdom with decreased mortality, shorter hospital stay, and less need for intensive care bed days. ${ }^{10}$ The surgical approach is usually a laparotomy following a period of resuscitation prior to surgery, if the condition of the patient allows it. At laparotomy, a thorough peritoneal lavage is performed and the site of the perforation is identified and sepsis is controlled. An attempt to look for and document any peritoneal disease present at that time is recommended; however, this can be difficult due to fecal peritonitis and widespread contamination. The site of perforation requires to be resected. If malignancy is suspected, or previously documented, optimal surgical oncological principles with must be followed with an appropriate lymphadenectomy performed. For a rightsided tumor, the decision on whether to perform an anastomosis or bring out a double-barrel stoma depends on the patient's condition and the level of contamination. In the case of left-sided tumors, the decision follows the same principles as in perforated benign disease. In a review of more than 900 patients undergoing emergency surgery for acute diverticulitis, Constantinides et $\mathrm{al}^{11}$ concluded that primary anastomosis had a lower mortality ( 7.4 vs. $15.6 \%$ ) compared with Hartmann's procedure, though, of course, sicker patients were more likely to have a Hartmann's such that this data should be interpreted with caution. In reality, the decision should be made on an individual case-by-case basis taking into account individual patient factors and surgical skill set available. A further factor to be considered in patients with malignancy is that an anastomotic leak not only increases morbidity and mortality but it will also significantly delay administration of systemic chemotherapy, potentially increasing the risk of systemic spread of disease.

Unfortunately, once a tumor has perforated, tumor cells are released into the peritoneal cavity with a risk of developing colorectal peritoneal metastasis (CPM). CPM carries a poor prognosis and used to be considered a terminal condition. However, over the last decade, advances in research and treatment pathways have dramatically changed this view. Carefully selected patients treated with a combination of cytoreductive surgery (CRS) and hyperthermic intraperitoneal chemotherapy (HIPEC) can have prolonged survival, and in some cases cured.

Patients who present with a perforated colorectal tumor should undergo full staging with a computed tomography (CT) of the chest, abdomen, and pelvis. If this has not been done during their acute presentation, then it should be performed once they have recovered from their acute septic episode. Their treatment should be coordinated through a multidisciplinary process, as the input of many specialties will be required. ${ }^{12}$

In the next sections of this article, we discuss the management of CPM which is more frequent following perforation of colorectal cancer.

\section{Spread of Colorectal Cancer}

Colon and rectal cancer can spread via the lymphatics into the corresponding lymph nodes, via blood vessels, directly to an adjacent organ or structure, and finally, through the transcoelomic route into the peritoneal cavity.

In the case of the lymphatic route, it tends to follow a pattern of first involving the lymph nodes closest to the primary tumor and with progression to more central nodes. Unfortunately, this progression does not always follow this ordered pattern.

In the case of hematogenous metastases, the liver is the organ most commonly affected with lung metastases, the second commonest. ${ }^{13}$ However, some patients have lung metastases without evidence of liver involvement implying, as with the lymphatic route, a more disorganized pattern of metastatic dissemination.

Direct spread can occur longitudinally, radially, and transversely. In the case of colorectal cancer, longitudinal spread can occur via tumor cells being shed into the lumen and then reimplanting distally. ${ }^{14}$ Radial spread can result in tumor growing directly into an adjacent structure such as the small bowel, duodenum, or ureter in the retroperitoneum, the abdominal wall, or the vagina.

Transcoelomic spread results from tumor cells released into the peritoneal cavity either by direct tumor perforation or rupture of an involved lymph node or vascular or lymphatic tumor into the peritoneal cavity.

\section{Peritoneal Carcinomatosis and the Concept of Colorectal Peritoneal Metastases}

Peritoneal involvement at the time of otherwise curative resection for a colorectal tumor is present in $\sim 5$ to $10 \%$ of patients, ${ }^{15,16}$ and 20 to $50 \%$ of all patients with CRC develop metachronous peritoneal involvement. ${ }^{17}$ Cells released into the peritoneal cavity are subject to the redistribution phenomenon, originally described to explain the effect of gravitational forces and intraperitoneal fluid dynamics on peritoneal disease distribution secondary to ruptured appendiceal neoplasms seen in pseudomyxoma peritonei (PMP). ${ }^{18}$ It is important to note here that PMP's biology (relative noninvasive) is generally much more favorable in most cases than that of CPM. In PMP, gravity allows pooling of peritoneal fluid in the pelvis and/or parabolic gutters, while clockwise flow of peritoneal fluid within the peritoneal cavity pushes cells into areas of peritoneal fluid reabsorption such as the omentum and subdiaphragmatic spaces, particularly the right diaphragm. In the case of PMP, the generally noninvasive biology usually results in visceral-sparing distribution of disease. In contrast, CPM with more invasive biology may infiltrate viscera at an earlier stage. However, selected cases of CPM, particularly in the early stages, may have a more contained distribution of peritoneal disease or even visceral-sparing distribution (especially with disease-free small bowel) and complete cytoreduction can be achieved resulting to improved outcomes and some cases even cure ${ }^{19}$ (-Fig. 1). There are several scoring systems such as the Peritoneal Carcinomatosis Index (PCI) (0-39), the Simplified Peritoneal Cancer Index (0-21), and the Seven Region Count from the Netherlands Cancer Institute, which all describe the 

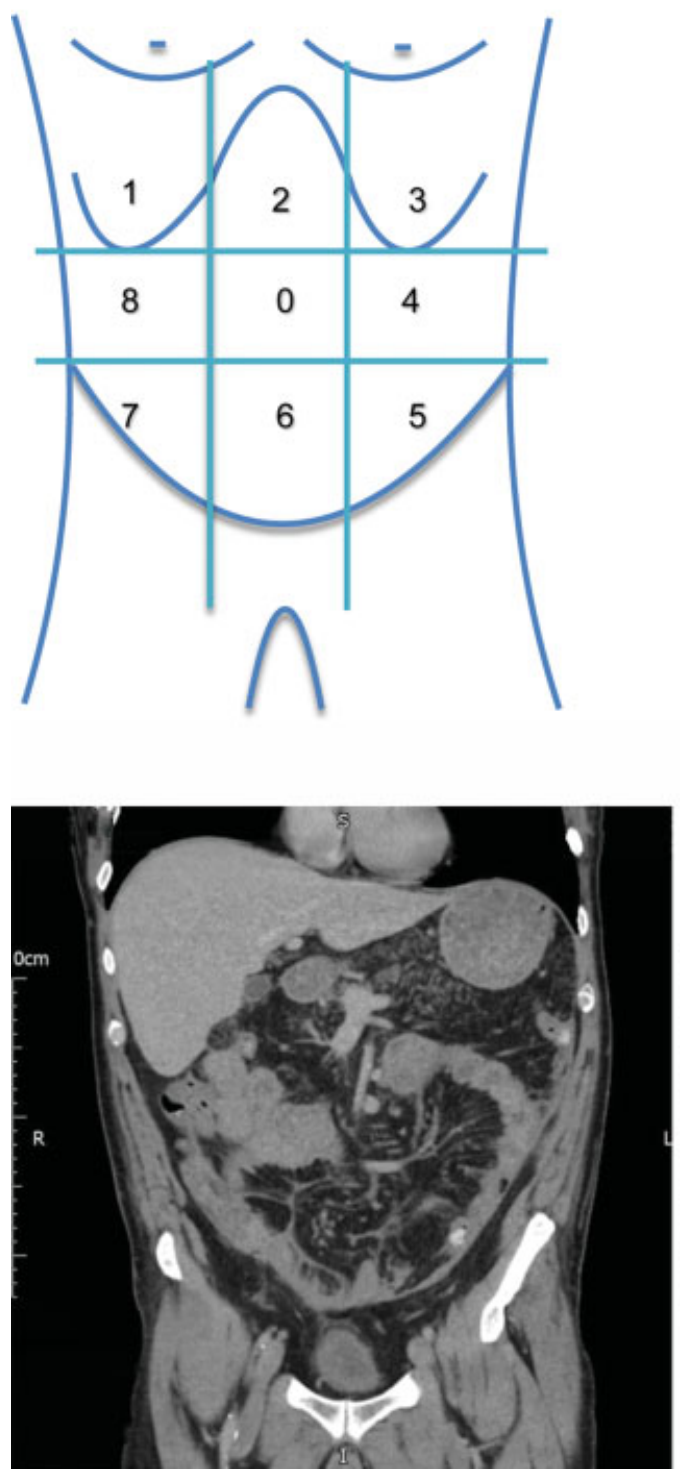

Fig. 1 Peritoneal Carcinomatosis Index: 0 to 8 correspond to nine sectors in the peritoneal cavity, while 9 to 13 split the small bowel into four sectors (upper jejunum, lower jejunum, upper ileum, and lower ileum). Every region is scored: $0=$ no tumor, $1=$ tumor $<5 \mathrm{~mm}, 2=$ tumor $<5 \mathrm{~cm}$, and $3=$ tumor $>5 \mathrm{~cm}$ or confluent disease.

distribution of disease within the peritoneal cavity. The most widely used is the PCI. In essence, $\mathrm{PCI}$ is computed by dividing the abdominal cavity into 13 areas or regions. Two transverse and two sagittal lines define the first nine areas. The upper transverse line is defined by connecting the two lowest points of the costal margin, and the lower by a line connecting the anterior iliac spines. The two sagittal lines divide the abdomen into three equal sectors. The nine regions are numbered clockwise starting at the right upper sector, with 0 being the central region (-Fig. 2). The remaining four areas refer to the upper and lower jejunum and upper and lower ileum. Each area is scored according to the size of the disease present $(0=$ no tumor, $1=$ tumor $<5 \mathrm{~mm}, 2=$ tumor $<5 \mathrm{~cm}$, and $3=$ tumor $>5 \mathrm{~cm}$ or confluent disease). The primary tumor is excluded from the PCI score.
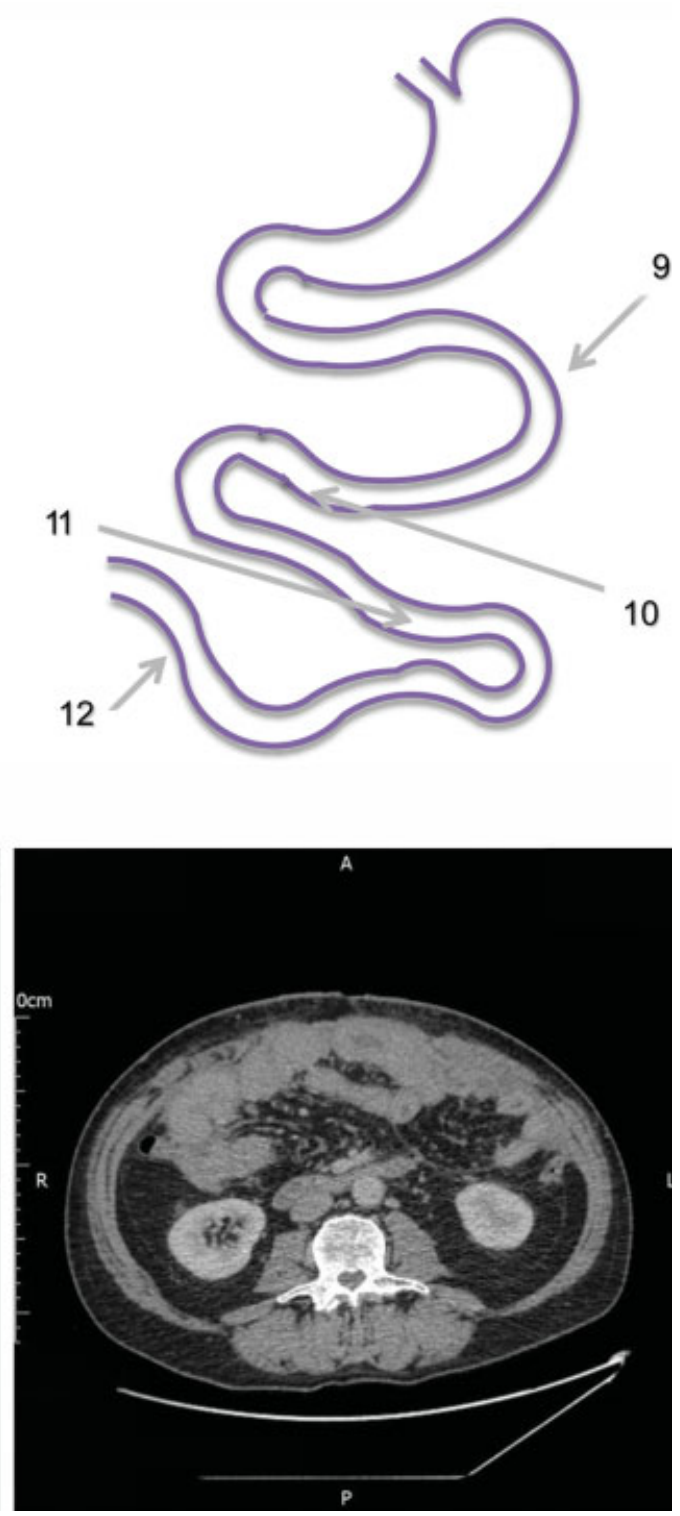

9

Patients with CPM who receive no treatment usually have a survival of less than 6 months. The most common cause of death is due to the indirect effect of peritoneal carcinomatosis such as bowel obstruction, fistulation, or malnutrition and generally not due to an overwhelming cancer burden. ${ }^{20}$ The most commonly used regimes are FOLFOX (5-FU combined with leucovorin and oxaliplatin) or FOLFIRI (5-FU, leucovorin and irinotecan), but unfortunately, the results are poor with a median overall survival of 10.4 to $15.2^{21,22}$

\section{Cytoreductive Surgery and Hyperthermic Intraperitoneal Chemotherapy}

The concept of complete removal of macroscopic disease and use of intraperitoneal chemotherapy was popularized by 

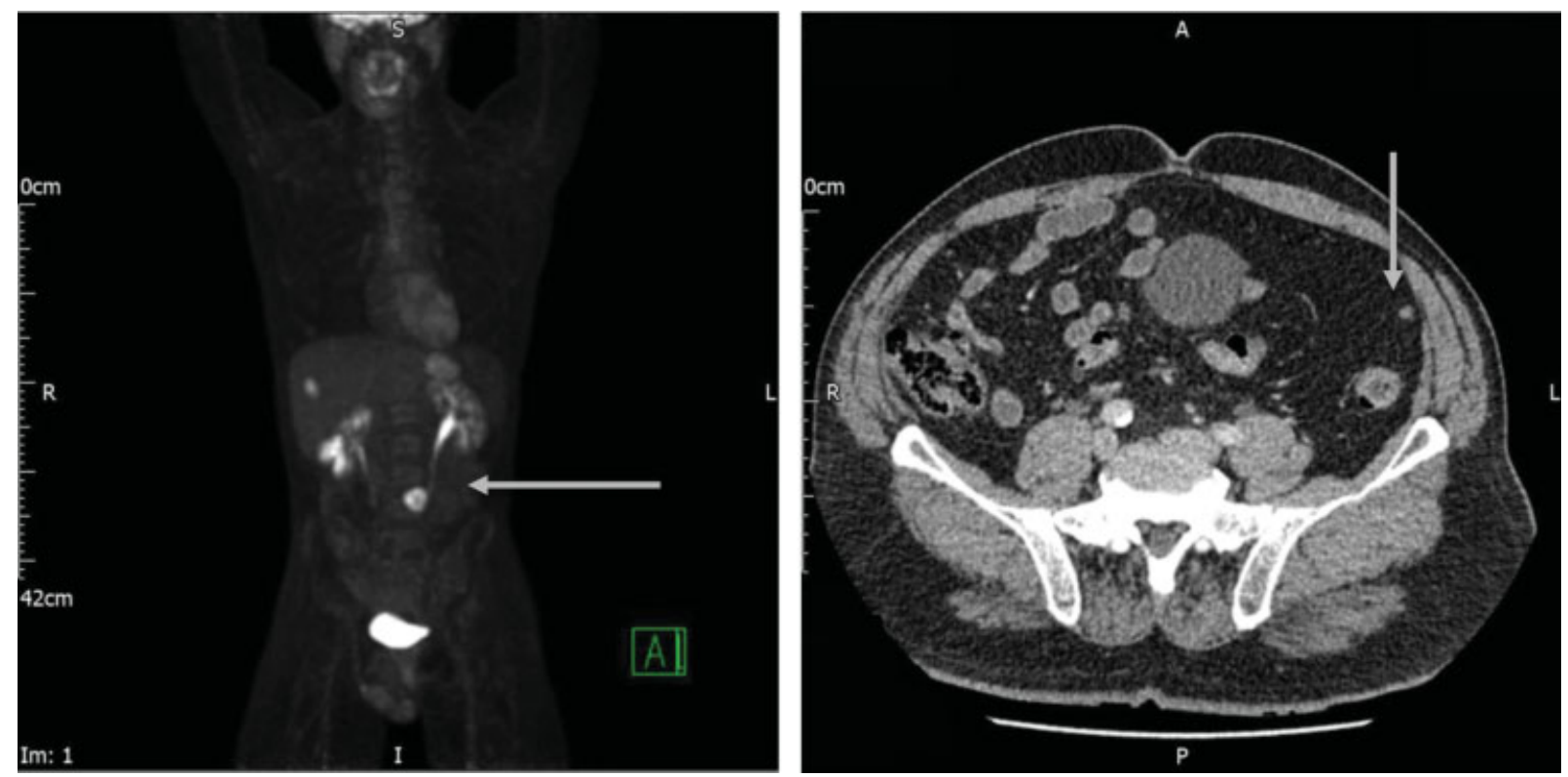

Fig. 2 CT scans of a patient with widespread peritoneal disease on the small bowel mesentery and serosa (top row images). PET-CT showing a solitary liver metastasis and solitary omental deposit (bottom right) and CT scan showing a single peritoneal deposit in the left paracolic gutter. $\mathrm{CT}$, computed tomography; PET, positron emission tomography.

Sugarbaker ${ }^{23}$ and others ${ }^{24}$ for the management of PMP with excellent results. The successful outcomes in PMP have led to the adoption of a similar strategy in the management of peritoneal disease from more biologically aggressive tumors such as colorectal cancer.

CRS and HIPEC consist of two components, namely, CRS aiming for complete macroscopic tumor removal combined with HIPEC. CRS aims to remove all visible diseases by targeted peritonectomies and multivisceral resection. The cytoreduction achieved is scored using the complete cytoreduction score $^{25}$ with CCO (no visible disease), CC1 $(<2.5 \mathrm{~mm})$, CC2 $(>2.5 \mathrm{~mm}$ but $<2.5 \mathrm{~cm})$, and CC3 $(>2.5 \mathrm{~cm})$. HIPEC involves administration of heated chemotherapy within the peritoneal cavity via an open or closed approach. In both approaches, chemotherapy is heated to 42 to $43^{\circ} \mathrm{C}$ and circulated by a perfusion machine using inflow and outflow catheters. The most commonly used chemotherapy agents are mitomycin C and oxaliplatin. There is no strong evidence favoring one or the other. Recently, the American Society of Peritoneal Surface Malignancies conducted a retrospective review ${ }^{26}$ of 15 international databases and looked at the use of mitomycin $\mathrm{C}$ and oxaliplatin reporting that the overall survival was similar for both drugs. In subset analyses of patients with a lower PCI, mitomycin $\mathrm{C}$ resulted in a better overall survival rate. However, this is a retrospective review with several limitations.

In 2003, Verwaal et $\mathrm{al}^{27}$ published a landmark randomized controlled trial comparing CRS and HIPEC (as experimental treatment) with systemic chemotherapy (5-FU and leucovorin) and palliative surgery if needed (as the standard treatment). Overall, 105 patients were randomized. The median survival rate in the systemic chemotherapy group was 12.6 months compared with 22.4 months in the CRS and HIPEC groups. Completeness of cytoreduction and extent of peritoneal involvement were independent predictors of better outcomes after CRS and HIPEC. The updated follow-up study published in $2008^{28}$ confirmed the initial results. In addition, the 5-year survival in the CRS and HIPEC groups with a complete cytoreduction was $45 \%$ which is comparable to that of hepatic metastasectomy for colorectal cancers. In 2010, Elias et $\mathrm{al}^{29}$ in a multicenter study reported on 523 patients who had CRS and HIPEC for CPM. Complete cytoreduction was achieved in $84 \%$ and this, together with a low PCI $(<19)$, absence of nodal disease and use of adjuvant chemotherapy were predictors of increased survival. The 5 -year overall survival was $27 \%$. In a more recent study, ${ }^{30}$ Elias et al (2015) reported overall survival of up to $72.4 \%$ at 5 years if the $\mathrm{PCI}$ was very low $(<5)$ and $38.7 \%$ if the $\mathrm{PCI}$ was between 6 and 15 .

The main issue is the associated morbidity (and mortality) and Mohamed et $\mathrm{al}^{31}$ in 2011 reported a range of morbidity of 12 to $67.6 \%$ and mortality of 0 to $9 \%$ in CRS and HIPEC highlighting that rates tend to decline with increasing experience as centers overcome the initial learning curve.

Careful and appropriate patient selection is essential to the success of CRS and HIPEC reducing the risks of exposing patients to a high-risk operation with small chances of benefit.

Patients should be fit enough to undergo a major operation and to tolerate major physiological cardiovascular challenges and possible complications. In addition, all efforts should focus on establishing the radiological PCI using CT or magnetic resonance imaging or through diagnostic laparoscopy if feasible. In the context of a patient presenting with a perforated tumor, the perforation should be addressed as described earlier. Thereafter, a period of systemic chemotherapy with a trial of time and reassessment, reimaging, and discussion with a peritoneal malignancy unit would be advisable depending on patients overall condition and response to systemic chemotherapy. Diagnostic laparoscopic may not be feasible in 
patients who have undergone emergency laparotomy for colorectal perforation and noninvasive imaging may be the only appropriate staging mechanism.

\section{The Concept of Second-Look Laparotomy}

In most patients, low-volume early peritoneal disease is asymptomatic and imaging has low sensitivity for CPM. However, these are the very group most likely to benefit from CRS and HIPEC. In 2011, Elias et al $^{32}$ reported a clinical trial encompassing a scheduled second-look laparotomy on asymptomatic patients deemed to be high risk of developing CPM based on the presence of localized and resected peritoneal disease at the index resection, a perforated tumor at presentation or ovarian metastasis (Krukenberg). Overall, 41 patients were included and after completion of adjuvant chemotherapy, postprimary resection underwent radiological staging. All 41 had normal scans and tumor markers and underwent a laparotomy between 6 and 12 months of their index operation. CPM was found in $60 \%$ of those that had initially localized and resected peritoneal disease, $37 \%$ of patients with perforated tumors and $62 \%$ in those with ovarian metastasis and all underwent CRS and HIPEC. In 2013, Honoré et $\mathrm{al}^{33}$ in a systematic review estimated the incidence of peritoneal metastases during follow-up postprimary resection without intraperitoneal chemotherapy at $70 \%$ if there was synchronous peritoneal nodules, $50 \%$ if the tumor had perforated and $20 \%$ if an adjacent organ or structure was involved or if there was tumor fistulation or obstruction. Randomized clinical trials are currently underway to address whether a second-look laparotomy in these high-risk groups results in similar incidence of CPM and if CRS and HIPEC improve survival or contribute to patient wellbeing or quality of life. In this context, a recent concept of "obstruction free survival" has been proposed as a possible benefit of CRS and HIPEC, even if cure is not achieved. ${ }^{34}$

\section{Conclusion}

About one-fifth of patients with colorectal cancer present as an emergency. The main emergency presentations are obstruction and perforation. There is overlap in some patients with both obstruction and perforation. The acute management of a patient with a perforated colorectal cancer is similar for any cause colonic perforation with the key priority to get the patient through the acute admission as safely and efficiently as possible. One of the major sequelae of a perforated colorectal malignancy is the development of CPM.

Awareness of the risks, and early detection of CPM with a strategy of CRS and HIPEC in carefully selected patients, results in good outcomes and cure in some cases and has been shown to be superior to what is generally rather ineffective systemic chemotherapy for CPMs.

\section{Conflict of Interest}

None.

\section{References}

1 Annual Report of the National Bowel Cancer Audit. Available at: https://www.nboca.org.uk. Accessed May 6, 2018

2 Survival of colorectal Cancer per stage, Cancer Research UK. Available at: http://www.cancerresearchuk.org. Accessed May 6, 2018

3 Sjödahl R, Franzén T, Nyström PO. Primary versus staged resection for acute obstructing colorectal carcinoma. Br J Surg 1992;79(07): 685-688

4 Runkel NS, Schlag P, Schwarz V, Herfarth C. Outcome after emergency surgery for cancer of the large intestine. Br J Surg 1991;78(02):183-188

5 Hogan J, Samaha G, Burke J, et al. Emergency presenting colon cancer is an independent predictor of adverse disease-free survival. Int Surg 2015;100(01):77-86

6 Baer C, Menon R, Bastawrous S, Bastawrous A. Emergency presentations of colorectal cancer. Surg Clin North Am 2017;97(03): 529-545

7 Yamamoto T, Kita R, Masui $\mathrm{H}$, et al. Prediction of mortality in patients with colorectal perforation based on routinely available parameters: a retrospective study. World J Emerg Surg 2015;10 (01):24

8 National Emergency Laparotomy Audit, United Kingdom. Available at: http://nela.org.uk/reports. Accessed May 14, 2018

9 Dellinger RP, Levy MM, Carlet JM, et al. Surviving Sepsis Campaign: international guidelines for management of severe sepsis and septic shock: 2008. Intensive Care Med 2008;34(01):17-60

10 Daniels R, Nutbeam T, McNamara G, Galvin C. The sepsis six and the severe sepsis resuscitation bundle: a prospective observational cohort study. Emerg Med J 2011;28(06):507-512

11 Constantinides VA, Tekkis PP, Athanasiou T, et al. Primary resection with anastomosis vs. Hartmann's procedure in nonelective surgery for acute colonic diverticulitis: a systematic review. Dis Colon Rectum 2006;49(07):966-981

12 Available at: https://www.nice.org.uk/Guidance/cg131. Accessed May 10, 2018

13 Cunningham D, Atkin W, Lenz HJ, et al. Colorectal cancer. Lancet 2010;375(9719):1030-1047

14 Umpleby HC, Fermor B, Symes MO, Williamson RC. Viability of exfoliated colorectal carcinoma cells. BrJ Surg 1984;71(09):659-663

15 Jayne DG, Fook S, Loi C, Seow-Choen F. Peritoneal carcinomatosis from colorectal cancer. Br J Surg 2002;89(12):1545-1550

16 Chu DZ, Lang NP, Thompson C, Osteen PK, Westbrook KC. Peritoneal carcinomatosis in nongynecologic malignancy. A prospective study of prognostic factors. Cancer 1989;63(02):364-367

17 Sadeghi B, Arvieux C, Glehen O, et al. Peritoneal carcinomatosis from non-gynecologic malignancies: results of the EVOCAPE 1 multicentric prospective study. Cancer: Interdisciplinary International. J Am Cancer Soc 2000;88(02):358-363

18 Sugarbaker PH. Pseudomyxoma peritonei. A cancer whose biology is characterized by a redistribution phenomenon. Ann Surg 1994;219(02):109-111

19 Moran BJ, Cecil TD. Treatment of surgically resectable colorectal peritoneal metastases. Br J Surg 2014;101(02):5-7

20 Hall B, Padussis J, Foster JM. Cytoreduction and hyperthermic intraperitoneal chemotherapy in the management of colorectal peritoneal metastasis. Surg Clin North Am 2017;97(03):671-682

21 Franko J, Shi Q Goldman CD, et al. Treatment of colorectal peritoneal carcinomatosis with systemic chemotherapy: a pooled analysis of north central cancer treatment group phase III trials N9741 and N9841. J Clin Oncol 2012;30(03):263-267

22 Klaver YL, Simkens LH, Lemmens VE, et al. Outcomes of colorectal cancer patients with peritoneal carcinomatosis treated with chemotherapy with and without targeted therapy. Eur J Surg Oncol 2012;38(07):617-623

23 Sugarbaker PH. Surgical treatment of peritoneal carcinomatosis: 1988 Du Pont lecture. Can J Surg 1989;32(03):164-170 
24 Moran B, Cecil T, Chandrakumaran K, Arnold S, Mohamed F, Venkatasubramaniam A. The results of cytoreductive surgery and hyperthermic intraperitoneal chemotherapy in 1200 patients with peritoneal malignancy. Colorectal Dis 2015;17(09):772-778

25 Portilla AG, Sugarbaker PH, Chang D. Second-look surgery after cytoreduction and intraperitoneal chemotherapy for peritoneal carcinomatosis from colorectal cancer: analysis of prognostic features. World J Surg 1999;23(01):23-29

26 Prada-Villaverde A, Esquivel J, Lowy AM, et al. The American Society of Peritoneal Surface Malignancies evaluation of HIPEC with mitomycin $C$ versus oxaliplatin in 539 patients with colon cancer undergoing a complete cytoreductive surgery. J Surg Oncol 2014;110(07):779-785

27 Verwaal VJ, van Ruth S, de Bree E, et al. Randomized trial of cytoreduction and hyperthermic intraperitoneal chemotherapy versus systemic chemotherapy and palliative surgery in patients with peritoneal carcinomatosis of colorectal cancer. J Clin Oncol 2003;21(20):3737-3743

28 Verwaal VJ, Bruin S, Boot H, van Slooten G, van Tinteren H. 8-year follow-up of randomized trial: cytoreduction and hyperthermic intraperitoneal chemotherapy versus systemic chemotherapy in patients with peritoneal carcinomatosis of colorectal cancer. Ann Surg Oncol 2008;15(09):2426-2432
29 Elias D, Gilly F, Boutitie F, et al. Peritoneal colorectal carcinomatosis treated with surgery and perioperative intraperitoneal chemotherapy: retrospective analysis of 523 patients from a multicentric French study. J Clin Oncol 2010;28(01): 63-68

30 Elias D, Faron M, Iuga BS, et al. Prognostic similarities and differences in optimally resected liver metastases and peritoneal metastases from colorectal cancers. Ann Surg 2015;261(01): 157-163

31 Mohamed F, Cecil T, Moran B, Sugarbaker P. A new standard of care for the management of peritoneal surface malignancy. Curr Oncol 2011;18(02):e84-e96

32 Elias D, Honoré C, Dumont F, et al. Results of systematic secondlook surgery plus HIPEC in asymptomatic patients presenting a high risk of developing colorectal peritoneal carcinomatosis. Ann Surg 2011;254(02):289-293

33 Honoré C, Goéré D, Souadka A, Dumont F, Elias D. Definition of patients presenting a high risk of developing peritoneal carcinomatosis after curative surgery for colorectal cancer: a systematic review. Ann Surg Oncol 2013;20(01):183-192

34 Moran BJ, Tzivanakis A. The concept of "Obstruction-Free Survival" as an outcome measure in advanced colorectal cancer management. Pleura and peritoneum 2018;3(01) 